\title{
¿QUÉ TAN PERTINENTE ES EL PROGRAMA DE INGENIERÍA INDUSTRIAL DE LA UNIVERSIDAD LIBRE?
}

\author{
How relevant is the industrial engineering program at Universidad Libre?
}

\author{
ANA M. RODRÍGUEZ L., ${ }^{1}$ DAVID A. RIVEROS O., ${ }^{2}$ RAMON M. CUBAQUE M. ${ }^{3}$
}

Recibido:11 de diciembre de 2020. Aceptado:16 de enero de 2021

DOI: http://dx.doi.org/10.21017/rimci.2021.v8.n15.a94

\begin{abstract}
Resumen
El propósito de este artículo es presentar los resultados del proyecto de investigación que tiene como objetivo evaluar la pertinencia del programa académico de ingeniería industrial de la Universidad Libre, el cual permitió determinar el grado de pertinencia social e industrial, tomando como base el perfil profesional definido en el PEP (Proyecto Educativo del Programa) vigente para el periodo 2018 - 2020 dando respuesta a los requisitos de acreditación del Acuerdo 02 de 2020 del CNA (Consejo Nacional de Acreditación). La información para este estudio fue recolectada a través de cuestionarios, como herramienta de investigación, los cuales se dirigieron a los empresarios vinculados a la institución, egresados del programa y a la comunidad que hace parte de la proyección social de la facultad.

En la evaluación se pudo concluir que el programa de Ingeniería Industrial cuenta un alto porcentaje de pertinencia, tanto social como industrial, valores que serán analizados en el presente artículo.
\end{abstract}

Palabras clave. Pertinencia; modelo; educación superior; evaluación de pertinencia

\begin{abstract}
The purpose of this article is to present the results of the research project that aims to evaluate the relevance of the academic program of industrial engineering of the free university, which allowed to determine the degree of social and industrial relevance, based on the professional profile defined in the PYP (Educational Project of the Program) in force for the period 2018 - 2020 responding to the accreditation requirements of Agreement 02 of 2020 of the CNA (National Accreditation Council). The information for this study was collected through questionnaires, as a research tool, which is addressed to the entrepreneurs linked to the institution, graduates of the program and the community that is part of the social projection of the faculty.

In the evaluation was possible to conclude that the Industrial Engineering program has a high percentage of relevance, both social and industrial, values that will be analyzed in this article.
\end{abstract}

Key words. Relevance; model; university education; relevance assessment.

\section{INTRODUCCIÓN}

L AS INSTITUCIONES de Educación Superior (IES) se enfrentan, hoy en día, a grandes retos relacionados con la evaluación de la pertinencia de sus programas académicos, entre los cuales se subra- yan con mayor relevancia, la adaptación a los cambios permanentes de necesidades que pueda tener una sociedad, una agremiación de industrias o una comunidad específica a la cual se le impacta, exigiendo estar a la vanguardia con las nuevas corrientes tecnológicas y cumplir con los requisitos

1 Proyectista, Universidad Libre, estudiante del programa Ingeniería Industrial

2 Proyectista, Universidad Libre, estudiante del programa Ingeniería Industrial

3 Proyectista, Universidad Libre, docente jornada completa del programa Ingeniería Industrial. Correo electrónico: ramoncubaque@yahoo.es ORCID: https://orcid.org/0000-0003-0794-2028 
esenciales de formación académica sin dejar de lado la preparación de profesionales integrales, competentes y con valores. El no responder adecuadamente a estos retos afecta la competitividad de los egresados de la institución [1].

Al ser una entidad de transición entre el mundo educativo y el mundo laboral es de vital importancia que las IES briden programas de formación académica pertinentes frente a las condiciones sociales y laborales entre otras, debido a que sus egresados juegan un papel trascendental dentro del desarrollo y evolución de la sociedad, siendo por aquel rol de las instituciones, que se hace necesario evaluar el nivel de pertinencia de cada uno de los programas académicos que ofrecen a la sociedad.

Es por esto que en la Universidad Libre, al ser una Institución de Educación Superior de alta calidad, se debe evaluar el nivel de pertinencia actual de su oferta académica de pregrado y posgrado, dentro de la cual se encuentra Ingeniería Industrial, programa del que se tienen escasas evidencias de su nivel de pertinencia social e industrial, partiendo de la falta de ajustes de actualización en la malla curricular desde su aprobación en el año 2005 , lo que ocasiona que no se pueda realizar un plan de acción que sea completamente efectivo en el momento de continuar mejorando la calidad del servicio que ofrece la institución.

A partir del diagnóstico y diseño de un modelo, se estableció el nivel de pertinencia social e industrial del programa de Ingeniería Industrial de la Universidad Libre, seccional de Bogotá, a través de la utilización de encuestas estructuradas para cada grupo de interés tales como los egresados del programa de las cohortes 2018 - 2020, los empresarios vinculados con la institución mediante el acercamiento que la práctica empresarial brinda a los estudiantes y la comunidad del entorno en la que el programa está impactado mediante los proyectos vinculados al desarrollo de la proyección social.

\section{Materiales y métodos}

La investigación realizada en este estudio es de carácter mixto debido a que tiene componentes desarrollados de forma cuantitativa y cualitativa
[2][3]. Cuantitativo porque se determinó el nivel de pertinencia social e industrial actual del programa mediante la captura, tabulación y análisis estadístico de la información obtenida, respecto a la calificación de aptitudes y la formación brindada por la Universidad Libre, y, cualitativo debido a que se desarrolló una categorización de aceptabilidad de la pertinencia del programa por parte de los interesados.

El contexto dónde se realizó la investigación fue el sector empresarial relacionado con la institución bajo el programa de práctica empresarial, la comunidad de impacto directo a través de organizaciones sin ánimo de lucro y la comunidad académica de la institución.

Dado que se conoce el tamaño de la población para cada uno de los grupos de interés en los que se aplicaron las encuestas, la fórmula aplicada para realizar el cálculo del tamaño de la muestra en cada uno de ellos es la siguiente [4]:

$$
n=\frac{N \cdot Z^{2} \cdot p \cdot q}{e^{2}(N-1)+Z^{2} \cdot p \cdot q}
$$

Donde:

$$
\begin{aligned}
\mathbf{N}= & \text { Tamaño de la población } \\
\mathbf{Z}= & \text { Nivel de confianza } \\
\mathbf{p}= & \text { Probabilidad de éxito, o proporción } \\
& \text { esperada } \\
\mathbf{q}= & \text { Probabilidad de fracaso }(1-\mathrm{p}) \\
\mathbf{e}= & \text { Precisión (error máximo admisible en tér- } \\
& \text { minos de proporción) }
\end{aligned}
$$

Teniendo un nivel de confianza $(Z)$ de $95 \%$, una proporción poblacional (p) supuesta de $50 \%$ dado que no se tiene conocimiento previo, y una precisión del 7\% para los egresados y la comunidad impactada y del $10 \%$ para los empresarios, se obtuvo que el tamaño de muestra correspondiste para cada grupo de interés es el siguiente. Tabla 1.

Tabla I. Tamaño de muestra por grupo de interés.

\begin{tabular}{|c|c|c|c|}
\hline & Egresados & Empresarios & Comunidad \\
\hline $\begin{array}{c}\text { Población } \\
(\mathrm{N})\end{array}$ & 219 & 159 & 80 \\
\hline $\begin{array}{c}\text { Muestra } \\
(\mathrm{n})\end{array}$ & 104 & 60 & 57 \\
\hline
\end{tabular}

Fuente: Los autores

Rev. Ingeniería, Matemáticas y Ciencias de la Información Vol. 8 / Núm. 15 / enero - junio de 2021; pág. 75-86 
Para la recolección de datos se desarrollaron encuestas siguiendo una estructura de preguntas abiertas y cerradas tanto dicotómicas como politómicas, las cuales fueron aplicadas a empresarios de diferentes sectores productivos con el fin de establecer si el enfoque y la formación brindada en el programa educativo de Ingeniería Industrial de la Universidad Libre tienen coherencia con el sector laboral donde la mayoría de sus profesionales se están desempeñando, y a su vez, determinar el grado en que satisfacen las necesidades de la industria. Con las encuestas aplicadas a los egresados del programa con un periodo de egreso no mayor a tres años se tenía como objetivo determinar el qué tan relacionados están la formación que brinda el programa con los conocimientos necesarios para el desarrollo de las actividades de los profesionales en la industria.

Las variables de investigación que se utilizaron en el desarrollo del trabajo y que permitieron establecer la pertinencia industrial [5] del programa salieron del análisis de los trabajos de los autores (autores de Antecedentes de campo) permitiendo identificar los siguientes (tabla II y III).

Tabla II. Indicadores de resultado: Sector Productivo.

\begin{tabular}{|l|l|}
\hline Indicador & Descripción \\
\hline Competitividad del profesional & $\begin{array}{l}\text { La inclinación de los empresarios por los egresados del programa de la } \\
\text { Universidad Libre }\end{array}$ \\
\hline Aceptación de practicantes & $\begin{array}{l}\text { Percepción del empleador frente al desempeño del practicante y su } \\
\text { preparación para el mundo laboral. }\end{array}$ \\
\hline Contratación de practicantes & $\begin{array}{l}\text { Periodo de tiempo por el que la empresa ha aceptado practicantes del } \\
\text { programa. }\end{array}$ \\
\hline Necesidad del sector productivo & $\begin{array}{l}\text { Contratación de practicantes al finalizar la practica } \\
\text { funciones en el entorno laboral. }\end{array}$ \\
\hline
\end{tabular}

Fuente: Los autores

Tabla III. Indicadores de resultado: Egresados.

\begin{tabular}{|l|l|}
\hline Indicador & Descripción \\
\hline Ubicación Laboral & Reconocer en qué tipo de empresa se encuentran los egresados del programa \\
\hline Satisfacción con la formación & $\begin{array}{l}\text { Porcentaje de titulados que, transcurrido un tiempo, se sienten satisfechos } \\
\text { con la formación recibida }\end{array}$ \\
\hline Inserción laboral & Conseguir empleo en el primer año de egreso. \\
\hline $\begin{array}{l}\text { Relación entre el plan de estudios y la } \\
\text { ocupación laboral }\end{array}$ & $\begin{array}{l}\text { Alineación del enfoque de la carrera con el sector económico en el que } \\
\text { laboran los egresados. }\end{array}$ \\
\hline $\begin{array}{l}\text { Relación entre los conocimientos } \\
\text { adquiridos y los que se necesitan en el } \\
\text { trabajo }\end{array}$ & $\begin{array}{l}\text { Formación brindada por la universidad cumple con las expectativas del } \\
\text { mercado laboral }\end{array}$ \\
\hline Liderazgo & Desarrollo de habilidades de liderazgo \\
\hline Emprendimiento & Egresados participes en la creación de una empresa. \\
\hline
\end{tabular}

Fuente: Los autores 
Se consolidaron y asignaron códigos de identificación a cada uno de los aspectos que se evaluaron del programa el cual está conformado por la letra "I" seguido de un número (Tabla III) lo que facilitó su análisis. Esta asignación no tuvo en cuenta ninguna distinción entre los elementos. Tabla IV.

Tabla IV. Codificación de los indicadores.

\begin{tabular}{|c|l|}
\hline Identificación & \multicolumn{1}{|c|}{ Nombre del indicador } \\
\hline 11 & Competitividad del profesional \\
\hline 12 & $\begin{array}{l}\text { Satisfacción con la preparación } \\
\text { técnica }\end{array}$ \\
\hline 13 & Aceptación de practicantes \\
\hline 14 & Contratación de practicantes \\
\hline 15 & Necesidad del sector productivo \\
\hline 16 & Ubicación laboral \\
\hline 17 & $\begin{array}{l}\text { Satisfacción del egresado con la } \\
\text { formación }\end{array}$ \\
\hline 18 & Inserción laboral \\
\hline 19 & $\begin{array}{l}\text { Relación entre el plan de estudios y } \\
\text { la ocupación laboral }\end{array}$ \\
\hline 20 & $\begin{array}{l}\text { Relación entre los conocimientos } \\
\text { adquiridos y los que se necesita en } \\
\text { el trabajo }\end{array}$ \\
\hline 21 & Habilidades de liderazgo \\
\hline 22 & Emprendimiento \\
\hline
\end{tabular}

Fuente: Los autores.

Para poder establecer el grado de importancia que tenía cada aspecto se evaluaron los indicadores haciendo uso de la herramienta investigativa Matriz de Vester [6], sin desconocer que el objetivo de esta herramienta es priorizar los problemas en una investigación, sin embargo, para efectos del desarrollo del trabajo tuvo como enfoque la priorización de factores entendiendo los criterios de calificación de la siguiente manera:

Tabla V. Rangos de calificación.

\begin{tabular}{|c|c|}
\hline Calificación & Relación de importancia \\
\hline 0 & No es importante \\
\hline 1 & Importancia baja \\
\hline 2 & Importancia media \\
\hline 3 & Importancia alta \\
\hline
\end{tabular}

Fuente: Los autores

Al terminar la evaluación de cada indicador se procedió a validar la matriz bajo el criterio de que la calificación máxima, en este caso " 3 ”, no superara el 30\% del total de las calificaciones; dado que se cumplió con este criterio se continuó con la ubicación de los factores en el plano cartesiano para realizar su clasificación teniendo en cuenta el siguiente razonamiento, haciendo una analogía de la clasificación de la Matriz de Vester (Tabla VI).

Tabla VI. Clasificación Matiz de Vester.

\begin{tabular}{|c|c|}
\hline Cuadrante & Descripción \\
\hline Crítico & $\begin{array}{l}\text { Determinante en el grado de } \\
\text { pertinencia del programa. }\end{array}$ \\
\hline Pasivos & $\begin{array}{l}\text { Tiene cierta relación o bajo } \\
\text { grado de afectación en la } \\
\text { pertinencia del programa. }\end{array}$ \\
\hline Activos & $\begin{array}{l}\text { Afectan el grado de } \\
\text { pertinencia del programa de } \\
\text { forma directa pero no son } \\
\text { determinantes en su } \\
\text { consecución. }\end{array}$ \\
\hline Indiferentes & $\begin{array}{l}\text { No afectan el grado de } \\
\text { pertinencia de un programa. }\end{array}$ \\
\hline
\end{tabular}

Fuente: Los autores.

La figura 1 permite reconocer en el plano cartesiano los cuadrantes antes mencionados.

La gráfica 1 mostrada a continuación fue realizada en el software de Excel, y permite identificar la ubicación de los indicadores.

Con la información obtenida al graficar los resultados de la matriz se reordenaron los indicadores, esta vez de forma jerárquica, empezando por el crítico, siguiendo con los activos, pasivos y por último los indiferentes [8]; asignando el grado de importancia expresado en porcentaje (Tabla VII).

Una vez organizados los indicadores, se realizaron y aplicaron los cuestionarios, tanto a egresados como empresarios, que permitieron obtener la información necesaria para establecer el nivel de pertinencia industrial del programa de Ingeniería Industrial de la Universidad Libre.

Para establecer el grado de pertinencia social [9][10], se tomaron las respuestas de la última pregunta del cuestionario realizado a los empresarios, dónde se indagaba sobre los tres principales valores que ellos consideraban que debería tener un profesional de ingeniería industrial, se ponderaron

Rev. Ingeniería, Matemáticas y Ciencias de la Información Vol. 8 / Núm. 15 / enero - junio de 2021; pág. 75-86 


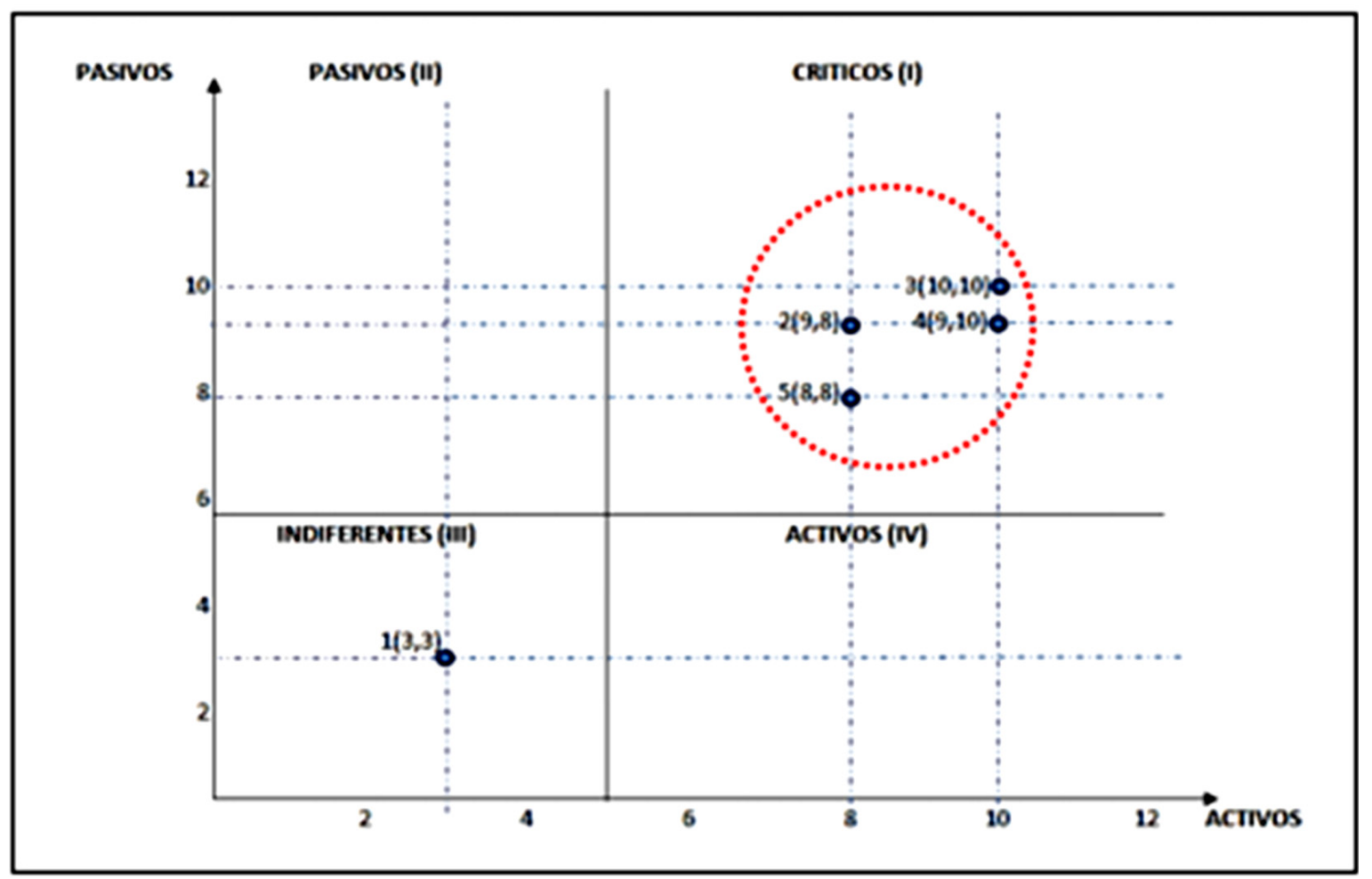

Fig. 1. Matriz de Vester identificando los cuadrantes [7]

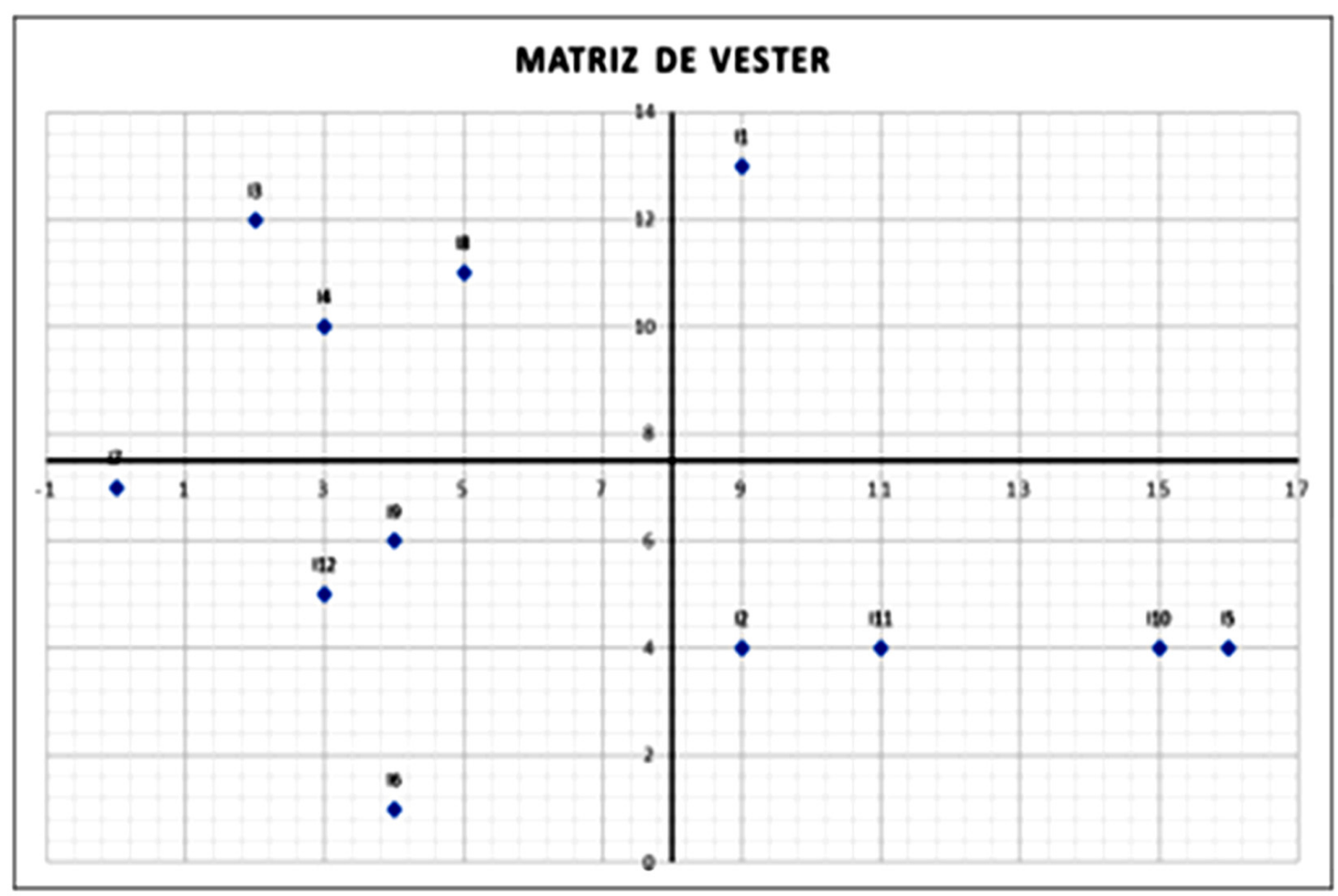

Gráfico 1. Gráfico Matriz de Vester. Fuente: Los autores. 
Tabla VII. Indicadores por importancia.

\begin{tabular}{|c|c|l|c|}
\hline Núm. & ID & \multicolumn{1}{|c|}{ Nombre del indicador } & Grado de importancia \\
\hline 1 & 11 & Competitividad profesional & $25 \%$ \\
\hline 2 & 15 & Necesidad del sector productivo & $15 \%$ \\
\hline 3 & 10 & $\begin{array}{l}\text { Relación entre los conocimientos adquiridos y los que se } \\
\text { necesitan en el trabajo }\end{array}$ & $9 \%$ \\
\hline 4 & 11 & Habilidades de liderazgo & $7.5 \%$ \\
\hline 5 & 12 & Satisfacción con la preparación técnica & $7.5 \%$ \\
\hline 6 & 13 & Aceptación de practicantes & $7.5 \%$ \\
\hline 7 & 14 & Contratación de practicantes & $2.5 \%$ \\
\hline 8 & 18 & Inserción laboral & $2.5 \%$ \\
\hline 9 & 17 & Satisfacción del egresado con la formación & $2.5 \%$ \\
\hline 10 & 12 & Emprendimiento & $2.5 \%$ \\
\hline 11 & 16 & Ubicación laboral & $2.5 \%$ \\
\hline 12 & 19 & Relación entre el plan de estudios y la ocupación laboral & \\
\hline
\end{tabular}

Fuente: Los autores.

y organizaron de tal forma que se permitieran establecer los más importantes y relevantes para la industria. Basados en esos resultados se realizó un nuevo formulario de preguntas dirigido a la comunidad del entorno sobre la cual el programa tiene un impacto directo dónde se solicitó que calificaran de uno a cinco qué tan desarrollados percibían estos valores en los estudiantes.

\section{Resultados}

Todos los cuestionarios necesarios para la ejecución de la investigación fueron realizados mediante la aplicación de Google forms y de igual forma se aplicaron de forma virtual logrando los siguientes resultados para cada indicador:

\section{A. Pertinencia Industrial}

Competitividad profesional.

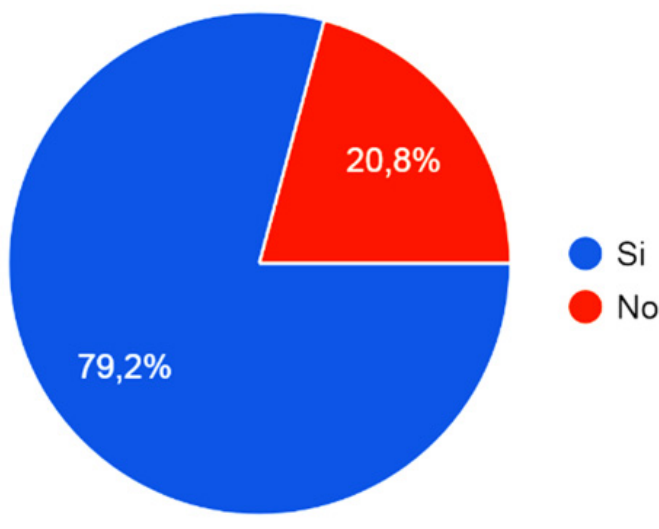

Gráfico 2. Competitividad profesional.
Al preguntar por la ocupación laboral de los graduados del programa de Ingeniería Industrial la investigación arrojó que la mayoría de ellos se encuentra económicamente activo.

Satisfacción con la preparación técnica.

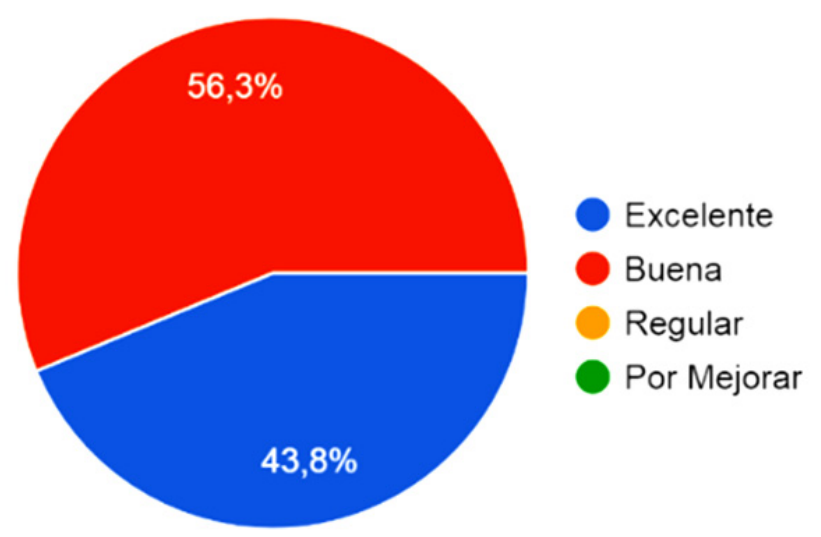

Gráfico 3. Satisfacción de los empresarios con la preparación técnica de los estudiantes.

La preparación académica que reciben los estudiantes del programa de Ingeniería cumple con las expectativas de la industria, así lo manifiesta la totalidad de los empresarios que han tenido un acercamiento a esta preparación dentro de la relación Universidad-Empresa que la práctica empresarial brinda.

Aceptaciones practicantes. 


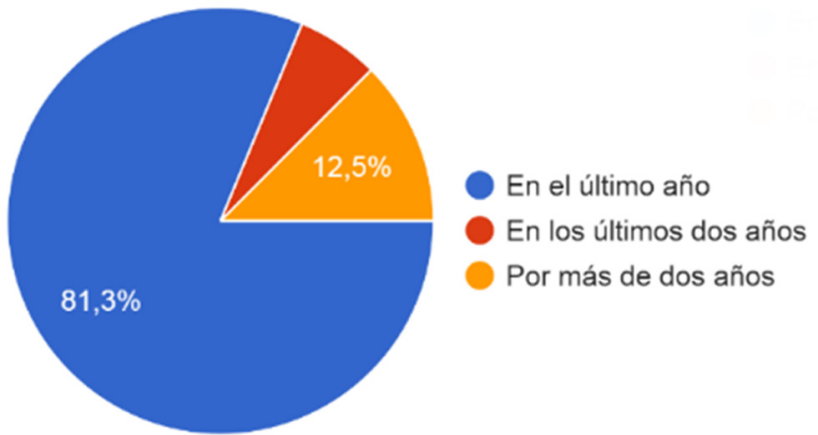

Gráfico 4. Aceptación de practicantes.

El cumplimiento de las expectativas académicas y las estrategias de expansión por parte de la Universidad para fortalecer esa parte de la triada Universidad-Empresa-Estado se ven reflejadas de buena manera dado que cerca del $\mathbf{8 1} \%$ de los empresarios ha optado por vincular por primera vez a practicantes del programa de Ingeniería Industrial en el último año, mientras que un $\mathbf{1 8 \%}$ de los empresarios ha desarrollado una preferencia por los practicantes del programa aceptándolos en sus compañías desde hace dos años o más.

Contratación de practicantes. Gráfica 5.

Pero que la formación académica brindada por la Universidad Libre cumpla con las expectativas de la industria no se manifiesta sólo en el interés de los empresarios por los estudiantes en periodo de práctica empresarial, sino también en la vinculación laboral de dichos estudiantes ya que cerca de $44 \%$ de los empresarios ha vinculado bajo contrato laboral a un practicante del programa de Ingeniería industrial en la compa-

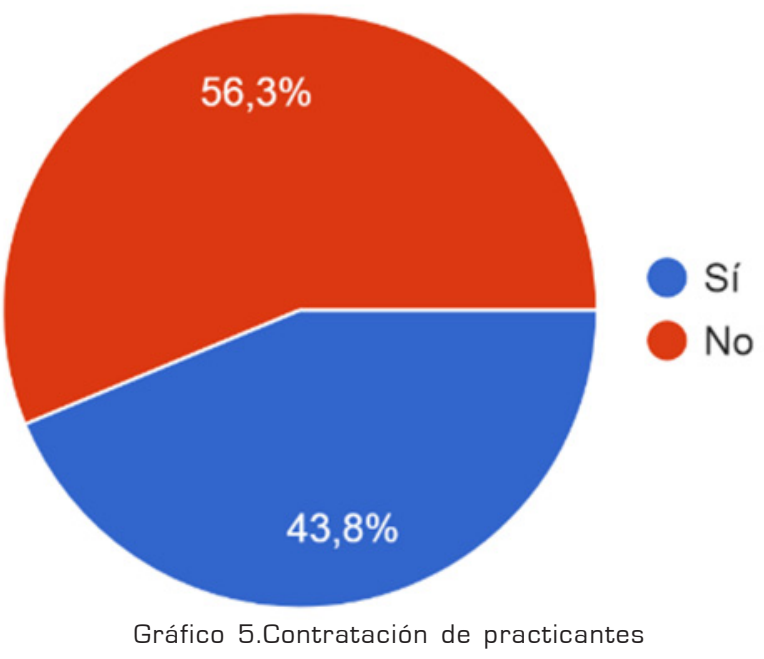

ñía después de haber culminado su periodo de práctica empresarial.

Necesidad del sector productivo. Gráfica 6.

Una evidencia de que la Universidad está brindando una formación alineada con las necesidades de la industria es el hecho de que los resultados de la tabla VIII muestran que cerca del $\mathbf{9 8 \%}$ de los empresarios consideran que las habilidades y conocimientos impartidos por la institución a los estudiantes del programa de Ingeniería Industrial son necesarios para el desarrollo de su vida profesional.

\section{Ubicación laboral. Gráfica 7.}

Apoyando el hecho de que la Universidad si se está brindando una formación que satisfaga las

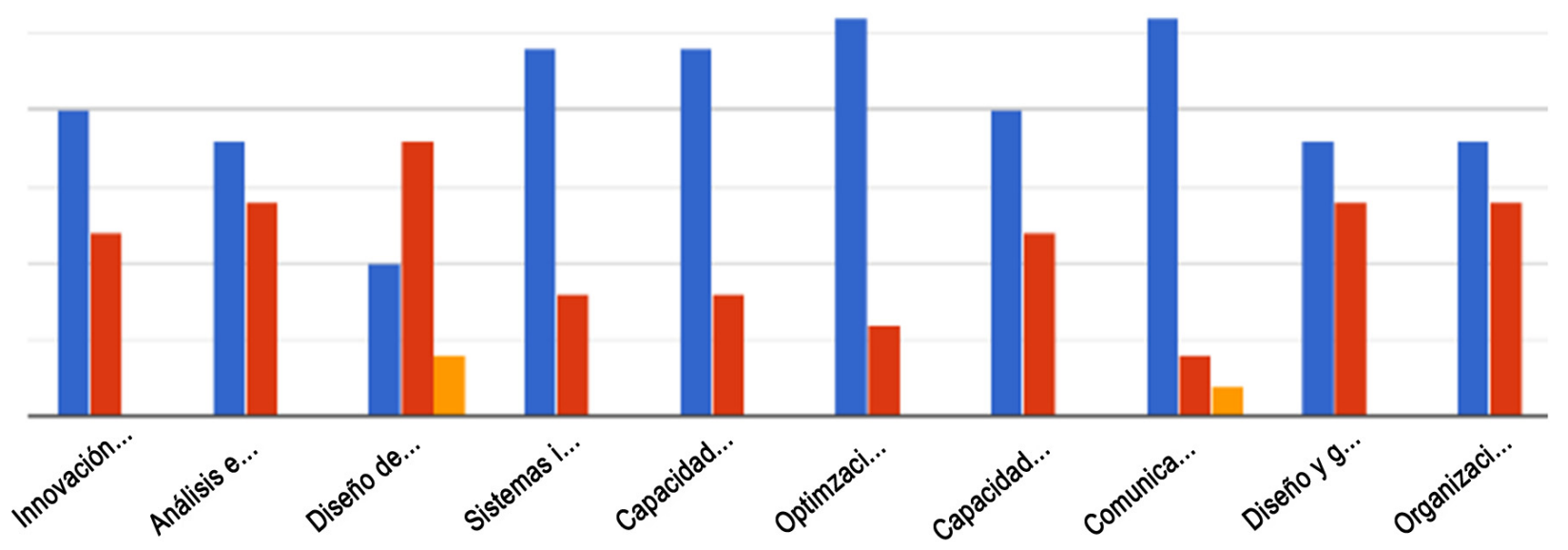

Gráfico 6. Calificación de las necesidades del sector productivo 
Tabla VIII. Resultados Indicador 5.

\begin{tabular}{|l|c|c|c|}
\hline \multicolumn{1}{|c|}{ Habilidades y conocimientos } & Muy necesaria & Necesaria & No necesaria \\
\hline Innovación de sistemas productivos y logísticos & $62,5 \%$ & $37,5 \%$ & . \\
\hline Analítica económico-administrativa & $56,2 \%$ & $43,8 \%$ & - \\
\hline Diseño de nuevos productos & $31,2 \%$ & $56,2 \%$ & $12,6 \%$ \\
\hline Sistemas integrados de gestión de calidad & $75 \%$ & $25 \%$ & - \\
\hline Capacidad de innovación en varias áreas & $75 \%$ & $25 \%$ & - \\
\hline Optimización y automatización de procesos & $81,2 \%$ & $18,2 \%$ & - \\
\hline Capacidad de construir y resolver problemas estadísticos & $62,5 \%$ & $37,5 \%$ & - \\
\hline Comunicación mediante un lenguaje gráfico y técnico & $81,2 \%$ & $12,5 \%$ & $6,3 \%$ \\
\hline Diseño y gestión de las operaciones & $75 \%$ & $25 \%$ & - \\
\hline Organización empresarial & $75 \%$ & $25 \%$ & - \\
\hline Porcentaje promedio & $67,5 \%$ & $30,6 \%$ & $1,9 \%$ \\
\hline
\end{tabular}

Fuente: Los autores

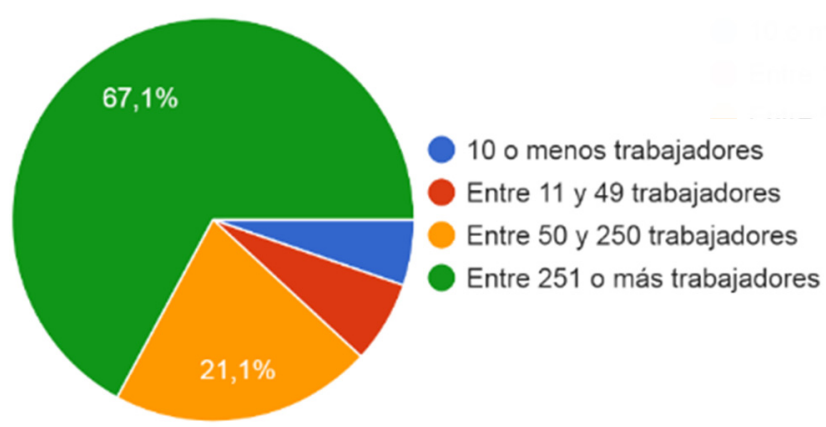

Gráfico 7. Ubicación laboral de los egresados.

necesidades de la industrial se encontró en la realización del estudio que aproximadamente el 67\% de los egresados está laborando en una empresa con presencia en más de una ciudad y/o país, según la clasificación del tamaño de empresa por número de empleados, y que alrededor del 21\% labora en una mediana empresa, mientras que una baja proporción labora en pymes.

Satisfacción del egresado con la formación. Gráfica 8.

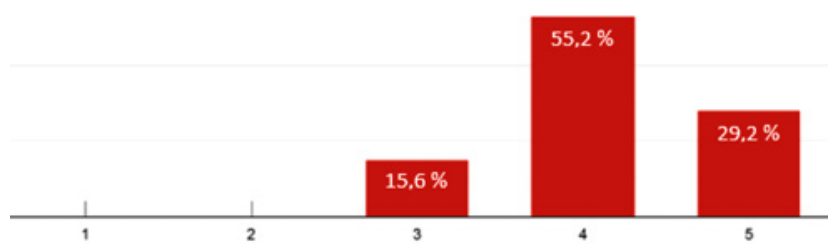

Gráfico 8. Satisfacción del egresado.
El nivel de satisfacción del egresado con la formación que recibió es otra muestra de este cumplimiento de expectativas, pero esta vez, vista desde el punto de vista del profesional pues cómo se muestra en el gráfico anterior cerca del $84 \%$ de los egresados se siente satisfecho con la formación que recibió por parte de la Universidad, mientras que se puede inferir que el $15 \%$ se siente indiferente respecto a la formación que recibió.

Inserción laboral. Gráfica 9.

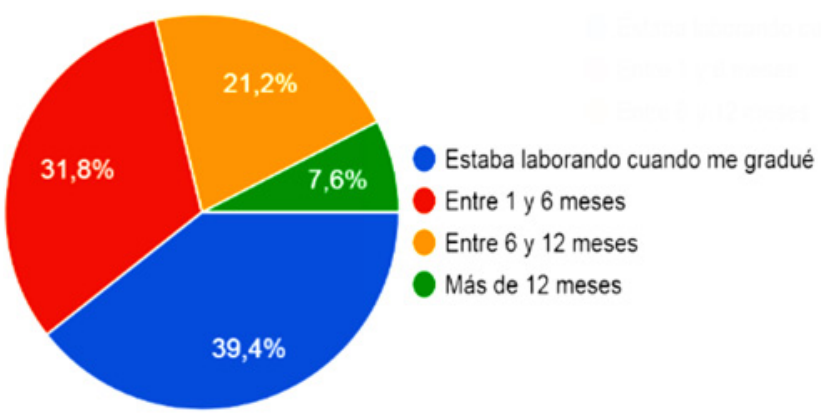

Gráfico 9. Inserción laboral de los egresados.

Parte de esta satisfacción se puede ver influenciada por el tiempo en que un egresado tarda en vincularse laboralmente en una compañía, pues, al preguntar por la velocidad de ocupación se encontró que más de la mitad de los egresados de una promoción $(53,0 \%)$ se ubican laboralmente en una empresa dentro de su primer año de egreso, mientras que el $39,4 \%$ ya se encontraban laborando al momento de graduarse. 
Relación entre el plan de estudios y la ocupación laboral. Gráfica 10.

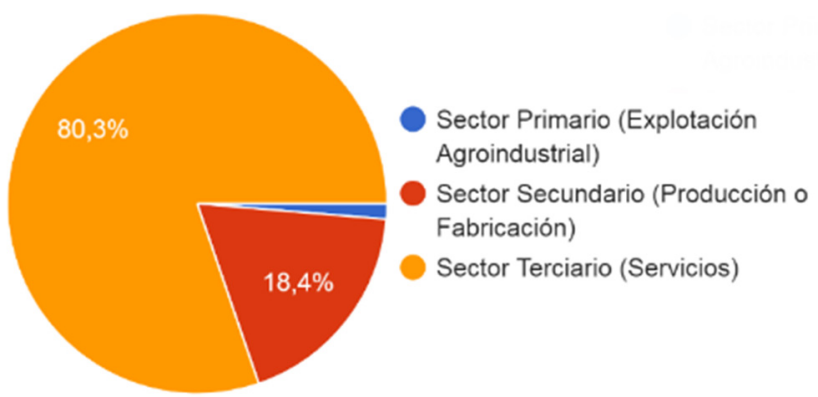

Gráfico 10. Relación entre el plan de estudios y la ocupación laboral.

Por otra parte, de los profesionales que están económicamente activos el 80,3\% de está laborando en una empresa del sector terciario, el 18,4\% está laborando en una empresa del sector secundario y el 1,3\% está laborando en el sector primario.

Relación entre los conocimientos adquiridos y los que se necesitan en el trabajo. Gráfica 11.

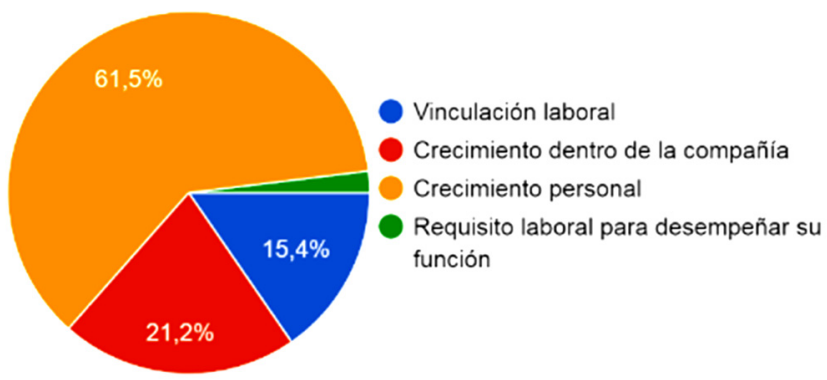

Gráfico 11.Cconocimientos adquiridos y necesarios en el trabajo.

A pesar de que la mayoría de los egresados labora en un sector diferente al enfoque que tiene el programa de Ingeniería Industrial tan sólo el 15,4\% de los egresados se vio en la necesidad de realizar un estudio académico adicional para vincularse laboralmente en una empresa.

Habilidades de liderazgo e ideas de Emprendimiento. Gráfica 12.

Por último, la investigación arrojó que cerca del $74 \%$ de los egresados ocupa un cargo administra-

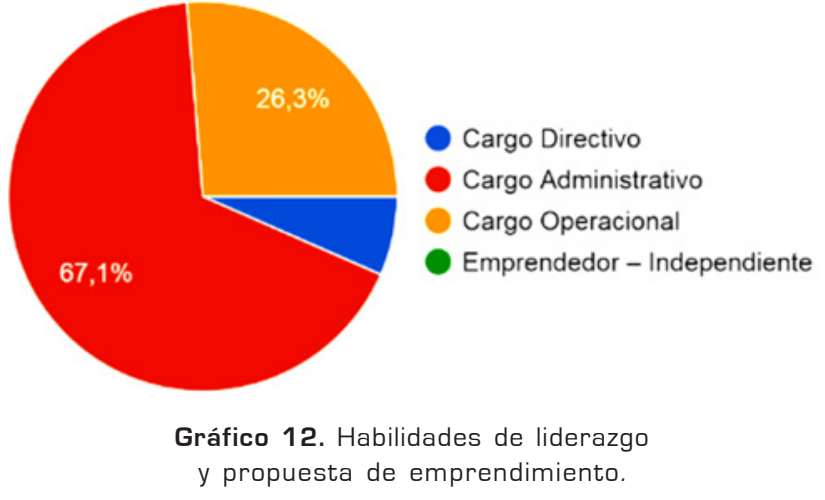

tivo o directivo en la empresa en la que labora; mientras que, por otra parte, no se evidenciaron propuestas de emprendimiento por parte de los profesionales.

En la tabla IX se consolidan los resultados de los indicadores anteriormente expuestos junto a la ponderación de cada uno; así como el nivel pertinencia industrial que tiene el programa expresado en porcentaje.

La información de la tabla anterior muestra que el programa de Ingeniería Industrial de la Universidad Libre, Seccional Bogotá, tiene un nivel de pertinencia Industrial del 88,9\%.

\section{B. Pertinencia Social}

Las habilidades duras y el desarrollo de conocimientos y habilidades no es el único componente importante en la formación de in profesional, los valores y habilidades personales también juegan un papel importante dentro del desempeño de cualquier persona, es por esto que para poder conocer aquellos valores y características personales que deben ser importantes en un profesional de Ingeniería industrial, se le solicitó a los empresarios que nombraran los tres principales valores que un profesional en este campo debe tener [11][12].

Dichas respuestas fueron ponderadas obteniendo como resultado las habilidades personales por las cuales se le preguntó a aquellas personas pertenecientes a la comunidad impactada por el proyecto de proyección social que desarrolla el programa, teniendo como resultados los siguientes datos. Gráfico 13. 
Tabla IX. Ponderación de factores.

\begin{tabular}{|c|l|c|c|c|}
\hline \multicolumn{5}{|c|}{ Ponderación de factores } \\
\hline Núm. & \multicolumn{1}{|c|}{ Nombre del indicador } & $\begin{array}{c}\text { Grado de } \\
\text { importancia }\end{array}$ & $\begin{array}{c}\text { Porcentaje } \\
\text { aceptable }\end{array}$ & $\begin{array}{c}\text { Resultados } \\
\text { encuesta }\end{array}$ \\
\hline 1 & Competitivdad del profesional & 25 & 85 & 79,2 \\
\hline 2 & Necesidad del sector productivo & 15 & 60 & 98,1 \\
\hline 3 & $\begin{array}{l}\text { Relación entre los conocimientos adquiridos y los que se } \\
\text { necesitan en el trabajo }\end{array}$ & 9 & 10 & 15,4 \\
\hline 4 & Habilidades de liderazgo & 9 & 70 & 73,7 \\
\hline 5 & Satisfacción con la preparación técnica & 9 & 75 & 100 \\
\hline 6 & Aceptación de practicantes & 7,5 & 60 & 81,3 \\
\hline 7 & Contratación de practicantes & 7,5 & 35 & 43,8 \\
\hline 8 & Inserción laboral & 7,5 & 60 & 53 \\
\hline 9 & Satisfacción del egresado con la formación & 2,5 & 65 & 84,4 \\
\hline 10 & Emprendimiento & 2,5 & 1 & 0 \\
\hline 11 & Ubicación laboral & 2,5 & 60 & 80,3 \\
\hline 12 & Relación entre el plan de estudios y la ocupación laboral & 2,5 & 60 & 18,4 \\
\hline Nivel de pertenencia industrial del Programa & & 80 & 88,9 \\
\hline
\end{tabular}

Fuente: Los autores.

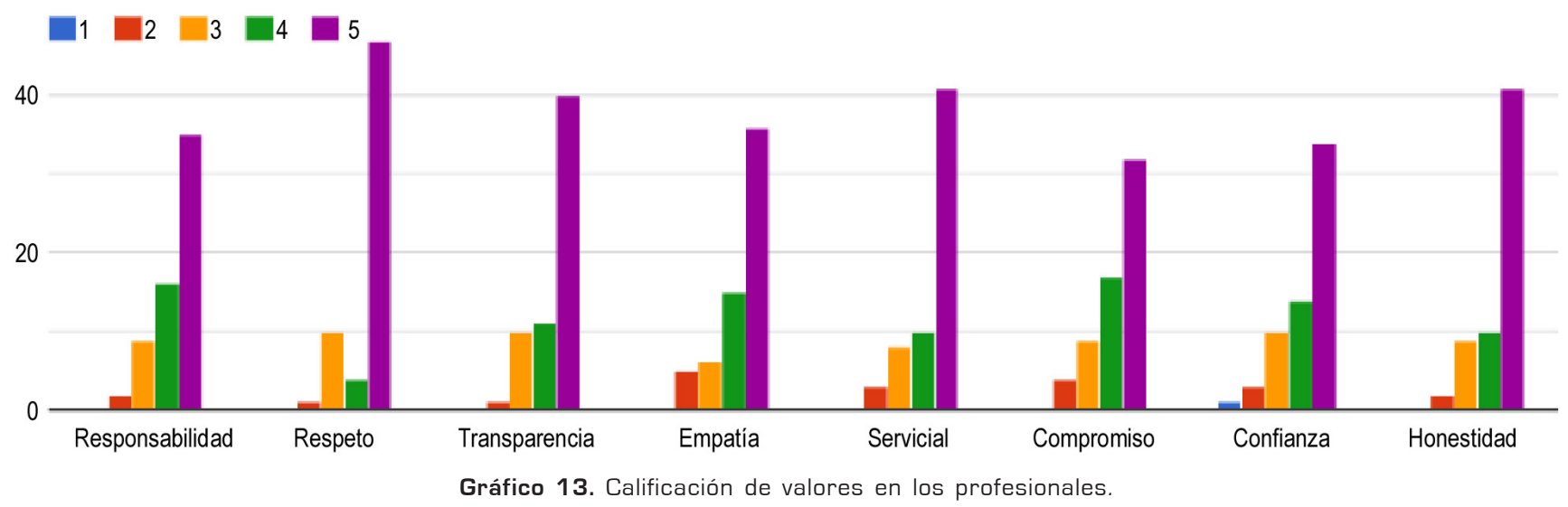

Los resultados de la anterior gráfica se muestran en la tabla $X$.

Los resultados obtenidos se evaluaron y consolidaron en la siguiente tabla, donde también se establece el nivel pertinencia social que tiene el programa en porcentaje. Tabla XI.

Con la información de la tabla anterior se establece que el programa de Ingeniería Industrial de la Universidad Libre, seccional Bogotá, tiene un nivel de pertinencia social del $81,5 \%$.

\section{Discusión}

Al realizar una revisión documental en las plataformas institucionales propias de la Universidad Libre, no se logró evidenciar estudios históricos dónde se evaluará el estado de la pertinencia del programa, lo más cercano que se encontró fueron varios estudios realizados como proyecto de grado cuyo enfoque era realizar un estudio del impacto de egresados, factor importante pero no suficiente para establecer la pertinencia de un programa educativo de una Institución de Educación 
Tabla X. Resultados Encuesta

\begin{tabular}{|l|c|c|c|c|c|}
\hline \multicolumn{1}{|c|}{ Habilidades personales } & $\mathbf{1}$ & $\mathbf{2}$ & $\mathbf{3}$ & $\mathbf{4}$ & $\mathbf{5}$ \\
\hline Responsabilidad & - & $3,2 \%$ & $14,3 \%$ & $25,4 \%$ & $57, \mathbf{1} \%$ \\
\hline Respeto & - & $1,6 \%$ & $15,9 \%$ & $6,3 \%$ & $76,2 \%$ \\
\hline Transparencia & - & $1.6 \%$ & $15,9 \%$ & $17,4 \%$ & $65,1 \%$ \\
\hline Empatía & - & $7,9 \%$ & $9,5 \%$ & $23,8 \%$ & $58,8 \%$ \\
\hline Servicial & - & $4,7 \%$ & $12,7 \%$ & $15,9 \%$ & $66,8 \%$ \\
\hline Compromiso & - & $6,3 \%$ & $14,3 \%$ & $17 \%$ & $52,4 \%$ \\
\hline Confianza & $1,60 \%$ & $4,7 \%$ & $15,9 \%$ & $22,2 \%$ & $55,6 \%$ \\
\hline Honestidad & - & $3,1 \%$ & $14,3 \%$ & $15,9 \%$ & $66,7 \%$ \\
\hline Porcentaje promedio & $1,60 \%$ & $4,1 \%$ & $14,1 \%$ & $19,2 \%$ & $62,3 \%$ \\
\hline
\end{tabular}

Fuente: Los autores

Tabla XI. Ponderación de Valores.

\begin{tabular}{|c|c|c|c|}
\hline Núm. & Valores & $\begin{array}{c}\text { Porcentaje } \\
\text { aceptabilidad }\end{array}$ & $\begin{array}{c}\text { Resultados } \\
\text { encuesta }\end{array}$ \\
\hline 1 & Responsabilidad & $80 \%$ & $82,5 \%$ \\
\hline 2 & Respeto & $80 \%$ & $82,5 \%$ \\
\hline 3 & Transparencia & $80 \%$ & $82,5 \%$ \\
\hline 4 & Empatía & $80 \%$ & $82,5 \%$ \\
\hline 5 & Servicial & $80 \%$ & $82,5 \%$ \\
\hline 6 & Compromiso & $80 \%$ & $82,5 \%$ \\
\hline 7 & Confianza & $80 \%$ & $79,3 \%$ \\
\hline 8 & Honestidad & $80 \%$ & $77,7 \%$ \\
\hline $\begin{array}{l}\text { Nivel de pertenencia } \\
\text { social del programa }\end{array}$ & $82,5 \%$ \\
\hline
\end{tabular}

Fuente: Los autores

superior. Se intuye que no se habían realizado estudios de la pertinencia del programa debido a la poca exigencia del Ministerio de educación en las evidencias al momento de evaluar un programa, así como la incidencia de que hay más factores que determinan el nivel de pertinencia diferente del impacto de los egresados.

Otro factor que hace parte de la discusión es que al realizar búsquedas en plataformas investigativas se encontró que se han realizado varios estudios de evaluación de pertinencia en diferentes niveles y con varios enfoques dirigidos a diversos programas a nivel nacional e internacional en otras universidades, sin embargo, no todos permitían tener una alineación y acercamiento a lo que se buscaba llegar con la realización del estudio, fue por esa razón que se seleccionaron algunos de ellos como base documental para el desarrollo y diseño del modelo de evaluación de pertinencia base, que al analizarse y simular su aplicación se fue adecuando a las necesidades y características de la Universidad Libre hasta llegar al modelo final que puede ser aplicado en los programas de educación superior de la institución.

Por medio de la virtualidad se puede llegar a tener más cobertura y alcance en el momento de capturar la información necesaria para llevar a cabo el estudio, a pesar de esto, se notó cierta resistencia por parte la población empresarial para responder el respectivo cuestionario diseñado para ellos entendiendo esto cómo una oportunidad de mejora en el fortalecimiento de las relaciones que tiene el programa con las diferentes empresas, puesto que se obtuvo una respuesta más rápida por parte de las compañías que tienen dos años o más de vinculación con la institución en contraposición con aquellas que llevan una relación menor a dos años. Desde otra perspectiva, tanto los egresados cómo la comunidad encuestada estuvo muy receptiva a responder la encuesta diseñada para cada uno de estos stakeholders, lo que puede traducirse en que las labores sociales realizadas por parte del programa están siendo bien recibidas y existe una relación de compañerismo entre sus profesionales.

Y como apreciación final de la discusión fue que al recolectar la información con las herramientas seleccionadas y analizar los datos capturados bajo el modelo de evaluación planteado en el trabajo, se obtuvieron los siguientes resultados de perti- 
nencia para el programa de Ingeniería Industrial de la Universidad Libre, seccional Bogotá, un nivel de pertinencia industrial igual a $88,9 \%$ y un nivel de pertinencia social equivalente al $81,5 \%$, catalogando ambos aspectos del programa como pertinente debido a que están por encima del porcentaje mínimo de aceptabilidad planteado por los autores, obteniendo así un nivel de pertinencia del $85,2 \%$ para el programa determinándolo como pertinente, lo que se traduce en el cumplimiento satisfactorio de los requisitos de acreditación de calidad expuestos en el Acuerdo 02 de 2020 del CNA.

\section{REFERENCIAS}

[1] MINISTERIO DE EDUCACIÓN NACIONAL, CONSEJO NACIONAL DE EDUCACIÓN SUPERIOR - CESU, CONSEJO NACIONAL DE ACREDITACIÓN. ACUERDO 02 DE 2020 - Por el cual se actualiza el Modelo de Acreditación en Alta Calidad. Disponible en: https://www.cna.gov.co/ 1741/articles-399640_A_CESU_02_2020_AMAC. pdf. 2020.

[2] Z. Pérez, Los diseños de método mixto en la investigación en educación: Una experiencia concreta. Recuperado de: https:/ / www.redalyc.org/pdf/ 1941/194118804003.pdf. 2011.

[3] A. Rustom, Estadística descriptiva, probabilidad e inferencia. Disponible en: http://repositorio. uchile.cl/bitstream/handle/2250/120284/ Rustom_Antonio_Estadistica_descriptiva.pdf?sequence $=1$

[4] W. Mendebhall, R. Beaver, B. Beaver, Introducción a la probabilidad y estadística. Disponible en: https://www.fcfm.buap.mx/jzacarias/cursos/ estad2/libros/book5e2.pdf. 2013.

[5] P. Almiocid, I. Montes y J. Vásquez, Un análisis factorial para evaluar la pertinencia de un programa académico desde la perspectiva de los graduados: un estudio de caso. Disponible en: https:/ / www.redalyc.org/articulo.oa?id=329027264003. 2009.
[6] J. Garcés, Análisis de la pertinencia del Programa Académico - Ingeniería Civil. Disponible en: http:/ /www.eafit.edu.co/institucional/calidad-eafit/ investigacion/investigacion/Documents / Analisis_de_la_pertinencia_del_programa_Ingenieria_ Civil-2011.pdf. 2011.

[7] F. Ramírez \& L. Tovar, Implementación de un sistema integrado de gestión Basado en ISO 9001:2008 y OHSAS 18001:2007 para la empresa Unión de tecnología electrónica - UNITEL LTDA. Recuperado de https://repository.unilibre.edu.co/ bitstream/handle/10901/9329/PROYECTO\% 20FINAL\%20def.pdf?sequence=1 2013.

[8] H. Parra Lopez, M.Vergel Ortega, J. Sanchez frank, J. Jacome Carrascal and J. Sepulveda, Disponible en: https://www.mineducacion.gov.co/1759/ articles-341895_archivo_pdf.pdf. 2020.

[9] D. Ramírez Carvajal, M. Gartner Isaza, J. Bernal Villegas, Á. Zapata Domínguez, F. Vallejo Cabrera, P. Prieto Pulido \& C. Langebaek Rueda, lineamientos para la acreditación de programas de pregrado Bogotá. Disponible en: http://cms. colombiaaprende.edu.co/static/cache/binaries/ articles-186359_pregrado_2013.pdf?binary_ rand $=7432.2013$.

[10] S. Tristancho y otros. Indicadores para evaluar la pertinencia social en la oferta académica de programas. Revista Logos, Ciencia \& Tecnología, 6(1), 142-155. ISSN: 2145-549X. Disponible en: https:/ / www.redalyc.org/articulo.oa?id=517751550012. 2014.

[11] A. C. Valdes Vento et al. Criterios de evaluación de la variable «pertinencia e impacto social» específicos para la carrera de Medicina. Educ Med Super. vol.23, n.3. Disponible en: http://scielo.sld.cu/ scielo.php?script $=$ sci_arttext\&pid=S0864-2141 2009000300005\&lng=es\&nrm=iso. 2009.

[12] N. E. Hamid Betancur, J. Alvarez Salazar, M. Torres Madronero Propuesta metodológica para medir la pertinencia y el impacto de programas de pregrado en ingeniería. Revista Educación en Ingeniería. p12(24), 97-100. Disponible en: https:/ / doi.org/10.26507/rei.v12n24.807. 2017. 